DOI: $10.4274 /$ gulhane.galenos.2022.49369

Gulhane Med J 2022;64:47-53

\title{
The prevalence of peripheral artery disease in older adults with chronic kidney disease
}

\author{
(1) Bilgin Bahadır Başgöz, (1) İlker Taşçı \\ University of Health Sciences Turkey, Gülhane Faculty of Medicine, Department of Internal Medicine, Ankara, Turkey
}

\section{Date submitted:}

27.10.2021

Date accepted:

30.01.2022

Online publication date:

15.03.2022

\section{Corresponding Author:}

Bilgin Bahadır Başgöz, M.D., Asst.

Prof., University of Health Sciences

Turkey, Gülhane Faculty of Medicine, Department of Internal Medicine,

Ankara, Turkey

bbbasgoz@gmail.com

ORCID:

orcid.org/0000-0002-5795-533X

Keywords: Peripheral arterial disease, chronic renal insufficiency, glomerular filtration rate, chronic kidney disease, older adults

Presented in: This study was presented as an oral presentation in $2^{\text {nd }}$ International and $12^{\text {th }}$ Academic Geriatrics Congress, 24-28 April 2019, Antalya, Turkey.

\begin{abstract}
Aims: Not only severe but also mild to moderate chronic kidney disease (CKD) is an independent risk factor for peripheral artery disease (PAD). In this study, we examined the prevalence of PAD among older adults with mild-to-moderate CKD.

Methods: The study was performed using the dataset of participants registered to a previous single-center, cross-sectional study that included non-institutionalized older adults aged 65 years or older in a geriatric outpatient setting. The subjects were patients with mild to moderate CKD. Ankle brachial index ( $\mathrm{BBI}$ ) was measured using a hand-held Doppler. PAD was diagnosed using an $\mathrm{ABI}$ value $<0.9$. The crude and adjusted prevalence of PAD were calculated.

Results: A total of 554 patients were included (age: 75.4 6.2 years; female: $67.3 \%$ ). PAD was detected by $8.2 \%, 27.1 \%, 60.0 \%$, and $4.7 \%$, in stage $1,2,3$ and 4 CKD, respectively, with significant difference in stage $2(p=0.003)$ and stage $3(p=0.011)$ CKD compared with the stage 1 disease. PAD was also more prevalent in patients with reduced estimated glomerular filtration rate (eGFR) (<60 mL/min/1.73 $\mathrm{m}^{2}$ vs. $\geq 60 \mathrm{~mL} / \mathrm{min} / 1.73 \mathrm{~m}^{2}: 19.6 \%$ vs. $\left.10.9 \%, \mathrm{p}=0.005\right)$. However, after adjustment for potential confounders, the increase in the prevalence of PAD in subjects with lower eGFR was no longer significant (Odds ratio: 1.48, 95\% confidence interval: 0.87-2.53, $\mathrm{p}=0.148)$.
\end{abstract}

Conclusions: This study showed a higher prevalence of PAD among older adults with mild-tomoderate CKD; however, the difference appears to depend on existing confounders.

\section{Introduction}

The occlusive atherosclerotic disease of the aorta and lower extremities are known as peripheral artery disease (PAD). PAD is classified as an atherosclerotic cardiovascular disease (ASCVD) with coronary artery disease (acute coronary syndrome, history of myocardial infarction, stable or unstable angina or coronary or other arterial revascularization), stroke, and transient ischemic attack (1-3).

The prevalence of PAD in the general population is around $3-10 \%(4,5)$. Also, independent of cardiovascular disease (CVD) risk factors, the incidence of the PAD progresses with age and reaches $15-20 \%$ in patients aged 70 years or older $(4,5)$. In the CAREFUL study, the first multicenter national study that investigated the prevalence of PAD in Turkey, subjects aged 70 years or older, or subjects aged 50-69 years with at least one cardiovascular risk factor were enrolled (6). The results of the study demonstrated that the prevalence of PAD was $20 \%$ in the study population, and $30 \%$ in patients aged 70 years or older (6). PAD accompanies complex chronic diseases of older age, as was shown in different types of dementia (7). 
Although the gold-standard test for the diagnosis of PAD is conventional or computed tomography angiography, the anklebrachial index $(\mathrm{ABI})$ measurement, a non-invasive, simpler, efficient, and cheaper diagnostic tool, became a preferable option in the diagnosis of PAD with sensitivity (Sen) and specificity (Spe) above $90 \%$ in all age groups $(8,9)$. Another alternative non-invasive technique used in the diagnosis of PAD is the arterial Doppler ultrasonography of the lower extremities (10). However, its preference is limited due to its poor performance in the diagnosis of infrapopliteal disease, inter-observer variability of the technique, and higher costs (10). Pulse wave velocity measurement, a non-invasive tool for the assessment of arterial stiffness, and Edinburgh Claudication Questionnaire, a screening tool for the detection of intermittent claudication were also studied as alternative methods for $A B I$, but results revealed that these tools cannot be used instead of each other $(11,12)$.

Chronic kidney disease (CKD) is prevalent among older adults and is associated with increased morbidity and mortality (13). Most older adults have a mild to moderate decrease in estimated glomerular filtration rate (eGFR) and a higher frequency of CKD due to the comorbidities (14). The overall prevalence of CKD in the general population is around $11 \%$, reaching greater than $40 \%$ after the $6^{\text {th }}$ decade (15).

Mild-to-moderate CKD is associated with a higher risk of ASCVD (16), which is more pronounced for older adults (17). However, most studies have focused on coronary artery disease (16). Only a few studies have been published on the association of PAD in patients with renal insufficiency (18). Thus, current knowledge on the frequency of PAD among older adults with CKD is considered limited. In this study, we investigated the prevalence of lower extremity PAD in older adults with mild-tomoderate CKD.

\section{Methods}

\section{Study population}

This study was performed using the dataset of participants registered to a previous single-center, cross-sectional study that included non-institutionalized older adults aged 65 years or older in a geriatric outpatient setting (19). Subjects receiving renal replacement therapy, having a history of lower extremity revascularization, recent major surgery, or extremity amputation, severely impaired in daily activities, residing in a nursing home, wheelchair bounded, or considered to have a short life expectancy due to malignancies or any other medical conditions and those who declined to participate were excluded from the original cohort. For the current analysis, subjects diagnosed with dementia were further excluded.

The institutional review board approved the study, and the participants provided written, informed consent when enrolled to the original study. The study protocol conforms to the Helsinki Declaration of 1975, as revised in 1983.

\section{Working protocol}

The basic characteristics of the subjects including age, gender, and self-reported education attained in years, current or past smoking status, and comorbidities including diabetes mellitus, hypertension, hypercholesterolemia, cardiovascular disease (CVD), coronary heart disease, stroke, anemia, hypothyroidism, vitamin B12 deficiency, and folate deficiency, body mass index (BMI), and laboratory results were available in the registry dataset.

\section{Calculation of GFR and determination of CKD stages}

Serum creatinine level was used to calculate eGFR (mL/ $\mathrm{min} / 1.73 \mathrm{~m}^{2}$ ) by using the Modification of Diet in Renal Disease (MDRD) study equation to determine the degree of kidney impairment (20). Stages of CKD was defined according to the 2012 Kidney Disease: Improving Global Outcomes (KDIGO) Clinical Practice Guideline as follows: stage 1 CKD: GFR $\geq 90$ $\mathrm{mL} / \mathrm{min} / 1.73 \mathrm{~m}^{2}$; stage 2 CKD: GFR 60 to $89 \mathrm{~mL} / \mathrm{min} / 1.73 \mathrm{~m}^{2}$; stage 3 CKD: GFR 30 to $59 \mathrm{~mL} / \mathrm{min} / 1.73 \mathrm{~m}^{2}$; stage 4 CKD: GFR 15 to $29 \mathrm{~mL} / \mathrm{min} / 1.73 \mathrm{~m}^{2}$; stage 5 CKD: GFR <15 mL/min/1.73 $\mathrm{m}^{2}$ or treatment by dialysis. However, individuals with CKD stage $5(n=2)$ were not included due to the very low number of subjects in the analyses because they were excluded from the original study.

\section{Measurement of $A B I$}

$\mathrm{ABI}$ was measured in an improved facility by the conventional method as previously described $(7,21-23)$. Briefly, patients were placed on a stretcher in the supine position with both arms slightly open. Two metal armrests of $30 \mathrm{~cm}$ width and $90 \mathrm{~cm}$ length were placed to the stretcher with an angle of $30^{\circ}$ degrees to support both arms. In addition, sponge rubbers were placed under the heels and elbows to slightly elevate the extremities from the surface below the heart level to allowing the operator to place the cuff ideally. Four fully calibrated and identical aneroid sphygmomanometers with Velcro cuffs (ERKA, D-83646, Bad Tölz, Germany) of $12 \mathrm{~cm}$ width and 29 to $42 \mathrm{~cm}$ length were wrapped on four extremities at the same time. After resting at least for five minutes in supine, measurements were performed using a handheld 8-MHz Doppler instrument (Hadeco, Kawasaki, Japan). The measurements were started from the right upper arm and followed by the right ankle left ankle and left upper arm. This cycle was performed twice. The first sound of blood flow heard during the deflation of the cuff for both brachial pulses in the upper arms and dorsalis pedis and tibialis posterior pulses in both ankles were recorded. The mean value of the two measurements was recorded as the result for the respective vessel. 


\section{Calculation and interpretation of $A B I$ results}

The $A B I$ was calculated based on the Trans-Atlantic InterSociety Consensus Document on Management of Peripheral Arterial Disease (TASC II) guidelines (9). The right and left ABIs were calculated separately by dividing the highest systolic blood pressures of each lower extremities (a. dorsalis pedis or a. tibialis posterior) to the systolic blood pressure of the brachial artery on the same side. Finally, the lower $A B I$ value on the left or right side was recorded as the final $A B I$ value. Individuals who had an $A B I$ value of 0.9 or lower were diagnosed with PAD. Besides, subjects with an $A B I$ value greater than $1.40(n=3)$ were excluded as it indicates noncompressible arteries that interfere with the correct assessment of the ABI.

\section{Statistical Analysis}

Results are displayed as the mean and standard deviation for normally distributed continuous variables. The normality of distribution was tested using the Shapiro-Wilk test. Categorical data are displayed as absolute numbers and a percentage of the total. The differences between the continuous variables were tested by the t-test. The chi-square test was used to compare the categorical variables. The Sen and Spe analysis was conducted to determine the effects of different stages of CKD on PAD. The study sample was divided into 3 tertiles according to the eGFR, as follows; tertile 1: eGFR $<49.4 \mathrm{~mL} / \mathrm{min} / 1.73 \mathrm{~m}^{2}(\mathrm{n}=138,25$ percentile), tertile 2: eGFR between $49.5-72.2 \mathrm{~mL} / \mathrm{min} / 1.73$ $\mathrm{m}^{2}(\mathrm{n}=277,50$ percentile $)$, tertile 3 : eGFR $>72.3 \mathrm{~mL} / \mathrm{min} / 1.73$ $\mathrm{m}^{2}$ ( $\mathrm{n}=139,75$ percentile). Tertile 1 had the lowest and tertile 3 had the highest eGFR. The analysis of continuous variables across tertiles was performed using one-way ANOVA or the Kruskal-Wallis test, and the analysis of categorical variables was performed using the chi-square test. Multivariable logistic regression analysis was used to test the independent association between the eGFR and PAD. The Hosmer-Lemeshow test was used to evaluate the goodness of fit of the model. Statistical significance was set at $p$ value $<0.05$. Statistical Package for the Social Sciences for Windows, version 26.0, IBM. Corp., Armonk, NY, 2019 was used for statistical analysis for all data.

\section{Results}

\section{Study population}

The study included 554 older adults (mean age: 75.4 \pm 6.2 years, women: 67.3\%). Women were significantly younger than men (mean age: $74.8 \pm 6.3$ vs. $76.8 \pm 5.8$; $p<0.001$ ). Basic demographics and clinical characteristics are given in Table 1. The mean eGFR was $62.0 \pm 20.6 \mathrm{~mL} / \mathrm{min} / 1.73 \mathrm{~m}^{2}$, and men have lower mean value than woman $\left(59.5 \pm 17.2 \mathrm{~mL} / \mathrm{min} / 1.73 \mathrm{~m}^{2} \mathrm{vs}\right.$. $63.2 \pm 22.0 \mathrm{~mL} / \mathrm{min} / 1.73 \mathrm{~m}^{2} ; \mathrm{p}=0.032$ ).

\section{Frequency of PAD according to the stages of CKD and tertiles}

In the whole study group, the frequency of patients with a 0.9 or lower $\mathrm{ABI}$ was $15.3 \%(n=85)$. The prevalence of PAD among

\begin{tabular}{|c|c|c|c|c|}
\hline & Total $(n=554)$ & Female $(n=373,67.3 \%)$ & Male (n=181, 32.7\%) & $\mathbf{p}$ \\
\hline Age, mean (SD) & $75.4(6.2)$ & $74.8(6.3)$ & $76.8(5.8)$ & $<0.001$ \\
\hline $65-74, \mathrm{n}(\%)$ & $277(50.0)$ & $203(36.6)$ & $74(13.4)$ & 0.001 \\
\hline $75-84, \mathrm{n}(\%)$ & $231(41.7)$ & $143(25.8)$ & $88(15.9)$ & 0.041 \\
\hline$>84, \mathrm{n}(\%)$ & $46(8.3)$ & $27(4.9)$ & $19(3.4)$ & 0.029 \\
\hline eGFR, mean (SD) & $62.0(20.6)$ & $63.2(22.0)$ & $59.5(17.2)$ & 0.032 \\
\hline BMI, mean (SD) & $29.8(5.6)$ & $30.8(5.9)$ & $27.7(4.3)$ & $<0.001$ \\
\hline Lower education, n (\%) & $390(70.5)$ & $316(57.1)$ & $74(13.4)$ & $<0.001$ \\
\hline Past/current smoking, n (\%) & $152(27.4)$ & $40(7.2)$ & $112(20.2)$ & $<0.001$ \\
\hline Diabetes mellitus, $n(\%)$ & $146(26.4)$ & $107(19.3)$ & $39(7.0)$ & 0.074 \\
\hline Hypertension, n (\%) & $432(78.0)$ & $300(54.2)$ & $132(23.8)$ & 0.046 \\
\hline Hypercholesterolemia, n (\%) & $224(40.4)$ & $160(28.9)$ & $64(11.6)$ & 0.090 \\
\hline Anemia, n (\%) & $92(16.6)$ & $78(14.0)$ & $14(2.6)$ & 0.007 \\
\hline Hypothyroidism, n (\%) & $62(11.5)$ & $48(8.9)$ & $14(2.6)$ & 0.056 \\
\hline Vitamin B12 deficiency, n (\%) & $168(30.3)$ & $107(19.3)$ & $61(11.0)$ & 0.228 \\
\hline Folate deficiency, $\mathrm{n}(\%)$ & $62(11.9)$ & $35(6.7)$ & $27(5.2)$ & 0.085 \\
\hline CVD, composite, $\mathrm{n}(\%)$ & $138(24.9)$ & $66(11.9)$ & $72(13.0)$ & $<0.001$ \\
\hline Coronary heart disease, $\mathrm{n}(\%)$ & $109(19.7)$ & $50(9.0)$ & $59(10.6)$ & $<0.001$ \\
\hline Stroke, n (\%) & $36(6.5)$ & $19(3.4)$ & $17(3.1)$ & 0.054 \\
\hline
\end{tabular}


subjects with stage 1, 2, 3 and 4 CKD was given in Table 2. Stage 1 and stage 4 CKD groups showed similar proportion of PAD $(8.2 \%$ vs. $7.9 \%, p=0.914$, and $3.0 \%$ vs. $4.7 \%, p=0.410$, respectively). The frequency of PAD was lower in stage 2 CKD (27.1\% vs. $44.1 \%, p=0.003)$, and higher in stage 3 CKD $(60.0 \%$ vs. $45.0 \%, p=0.011$ ) (Table 2). Also, the prevalence of PAD was higher in patients with a reduced $\left(<60 \mathrm{~mL} / \mathrm{min} / 1.73 \mathrm{~m}^{2}\right)$ vs. higher eGFR (19.6\% vs. $10.9 \%, p=0.005)$. However, after adjustment for potential confounders (age, gender, smoking, diabetes mellitus), the increase in the prevalence of PAD in subjects with reduced eGFR was no longer significant [OR: 1.48, 95\% confidence interval $(\mathrm{Cl})$ : 0.87-2.53, $\mathrm{p}=0.148$ ] (HosmerLemeshow test, $p=0.890)$.

The frequency of PAD between the eGFR tertiles was significantly different (tertile $1: 36.5 \%$, tertile $2: 48.2 \%$, and tertile 3: $15.3 \%, p=0.010$ ) (Table 2). The comparison of patients in the lowest and highest tertiles showed that the mean $A B I$ value was significantly higher (T1: $0.99 \pm 0.19$ vs. T3: $1.07 \pm 0.14, p<0.001$ ), and the prevalence of PAD was significantly lower (T1: $22.5 \%$ vs. T3: $9.4 \%, p=0.003$ ) in the highest tertile (Table 3).

The Sen and Spe of each CKD stages and reduced eGFR to detect PAD were as follows; stage $1 \mathrm{CKD}=8.2 \%$ Sen and $92.1 \%$ Spe; stage $2 \mathrm{CKD}=27.1 \%$ Sen and $55.9 \%$ Spe; stage 3 CKD $=60.0 \%$ Sen and $55.0 \%$ Spe; stage 4 CKD=4.7\% Sen and
97.0\% Spe, and eGFR $<60 \mathrm{~mL} / \mathrm{min} / 1.73 \mathrm{~m}^{2}=64.7 \%$ Sen and $57.0 \%$ Spe.

\section{Discussion}

The results of this study showed that an eGFR value below $<60 \mathrm{~mL} / \mathrm{min} / 1.73 \mathrm{~m}^{2}$ is associated with a higher frequency of PAD among older patients. Also, the comparison of subjects in the lowest and highest eGFR tertiles showed that PAD was more prevalent, and the mean $A B I$ value was lower in the lowest eGFR tertile. However, adjusted for age, gender, smoking status, and diabetes mellitus, the difference in the prevalence of PAD patients with eGFR below and above $60 \mathrm{~mL} / \mathrm{min} / 1.73 \mathrm{~m}^{2}$ became saturated.

The risk of ASCVD associated ischemic events is higher in CKD patients than in individuals with preserved kidney function (24). The PAD, similar to other ASCVD, is also more frequent in CKD patients than in the general population (25). Although the association between severe CKD and PAD in the general population is well established (25), the data related to the association of mild to moderate CKD and PAD among older adults are limited. Most of the studies that focused on the association of ASCVD and reduced eGFR among older adults excluded patients with PAD $(17,26)$. Either the rate of PAD patients in the study cohort was limited (27), or the participants were mostly younger than 65 years in other studies (18).

\begin{tabular}{|c|c|c|c|c|}
\hline & Total $(n=554)$ & $\begin{array}{l}\text { Peripheral artery disease } \\
(n=85,15.3 \%)\end{array}$ & $\begin{array}{l}\text { No peripheral artery disease } \\
(n=469,84.7 \%)\end{array}$ & $\mathbf{p}$ \\
\hline Stage 2 CKD, n (\%) & $230(41.5)$ & $23(27.1)$ & $207(44.1)$ & 0.003 \\
\hline Stage 3 CKD, n (\%) & $262(47.3)$ & $51(60.0)$ & $211(45.0)$ & 0.011 \\
\hline Tertile 1 & $138(24.9)$ & $31(36.5)$ & $107(22.8)$ & \multirow{3}{*}{0.010} \\
\hline Tertile 2 & $277(50)$ & $41(48.2)$ & $236(50.3)$ & \\
\hline Tertile 3 & $139(25.1)$ & $13(15.3)$ & $126(26.9)$ & \\
\hline \multicolumn{5}{|c|}{$\begin{array}{l}\text { Stage } 1 \text { CKD: eGFR } \geq 90 \mathrm{~mL} / \mathrm{min} / 1.73 \mathrm{~m}^{2} \text {, stage } 2 \text { CKD: eGFR between } 89-60 \mathrm{~mL} / \mathrm{min} / 1.73 \mathrm{~m}^{2} \text {, stage } 3 \mathrm{CKD} \text { : eGFR between } 59-30 \mathrm{~mL} / \mathrm{min} / 1.73 \mathrm{~m} \mathrm{~m}^{2}, \text { stage } 4 \\
\text { CKD: eGFR between } 29-15 \mathrm{~mL} / \mathrm{min} / 1.73 \mathrm{~m}^{2} \text {, tertile } 1 \text { : eGFR }<49.4 \mathrm{~mL} / \mathrm{min} / 1.73 \mathrm{~m}^{2} \text {, tertile } 2: \text { eGFR between } 49.5-72.2 \mathrm{~mL} / \mathrm{min} / 1.73 \mathrm{~m}{ }^{2} \text {, tertile } 3: \text { eGFR }>72.3 \mathrm{~mL} / \\
\mathrm{min} / 1.73 \mathrm{~m}^{2} \text {. Significant p values are in bold. } \\
\text { CKD: Chronic kidney disease, eGFR: Estimated glomerular filtration rate }\end{array}$} \\
\hline
\end{tabular}

Table 3. Comparison of the frequency of peripheral artery disease and the mean ankle brachial index values in subjects with reduced and preserved estimated glomerular filtration rate and in lowest and highest tertiles

\begin{tabular}{|c|c|c|c|}
\hline & \multicolumn{2}{|c|}{ Estimated glomerular filtration rate } & \multirow[b]{2}{*}{$\mathbf{p}$} \\
\hline & Reduced $(n=280)$ & Preserved $(n=274)$ & \\
\hline PAD, n (\%) & $55(19.6)$ & $30(10.9)$ & 0.005 \\
\hline PAD, n (\%) & $31(22.5)$ & $13(9.4)$ & 0.003 \\
\hline $\mathrm{ABI}$, mean (SD) & $0.99(0.19)$ & $1.07(0.14)$ & $<0.001$ \\
\hline
\end{tabular}


In a study conducted in Spain with 102 older adults with CKD, patients with reduced eGFR $\left(<60 \mathrm{~mL} / \mathrm{min} / 1.73 \mathrm{~m}^{2}\right)$ were diagnosed with PAD by $32 \%$, markedly higher than the prevalence of PAD in Spanish population without CKD (6.9\%) (28). However, the authors performed only unadjusted analyses. Although we diagnosed more patients with PAD among CKD patients with a reduced eGFR $\left(<60 \mathrm{~mL} / \mathrm{min} / 1.73 \mathrm{~m}^{2}\right)$ in crude comparisons, the difference was no longer significant in the adjusted analysis.

In a longitudinal study conducted with 4893 subjects aged 65 years or older, participants were followed up for a mean of 4.33 years to determine the relationship between baseline kidney function and the development of ASCVD (29). The authors identified that $25.1 \%(n=1229)$ of the participants were diagnosed with ASCVD during follow-up, but only 1.5\% $(n=18)$ were PAD. The authors also reported that each $10 \mathrm{~mL} / \mathrm{min} / 1.73$ $\mathrm{m}^{2}$ decline in eGFR was associated with an increased risk of ASCVD, including PAD. However, the number of PAD diagnoses in that study was limited. Also, the authors did not perform a subgroup analysis with PAD patients to investigate whether the increased risk of ASCVD due to a decrease in eGFR could have been similarly attributed to the PAD patients or not.

According to results of the National Health and Nutrition Examination Survey of 1999-2000, the prevalence of PAD among patients with an eGFR $<60 \mathrm{~mL} / \mathrm{min} / 1.73 \mathrm{~m}^{2}$ vs. higher was found $24 \%$ vs. $3.7 \%$, respectively (25). Moreover, the association of PAD and CKD remained significant after adjustment for potential confounders, which contrasts with our results of frequency analysis as the difference in the prevalence of PAD among subjects with low vs. preserved eGFR was not significant controlling for potential covariates. The exclusion of patients with stage $5 \mathrm{CKD} /$ renal replacement therapy and significantly older age of the study population in our study may explain the difference between the two studies $(30,31)$.

In a prospective follow-up study by Wattanakit et al. (26), a total of 14280 participants with a mean age of 54 years were followed up for a mean of 13.1 years to investigate whether the level of kidney function was an independent risk factor for PAD. Age and gender-adjusted relative risk (RR) for PAD was 1.04 (95\% Cl: 0.91-1.18) for those with stage 1 and 2 CKD, and 1.82 (95\% Cl: 1.34-2.47) for those with stage 3 and 4 CKD. After adjustment for confounders of ASCVD development, although the RR was reduced, patients with CKD were still at an increased risk of incident PAD (RR of 1.56; $\mathrm{Cl}$ : 1.13-2.14). The findings suggested an inverse correlation between the risk of PAD and kidney functions across all stages of CKD. We were unable confirm that finding among mild-to-moderate CKD patients in our study. On the explanation may be the unexpectedly high frequency of PAD in patients with preserved renal functions due to the advanced age of our cohort.
PAD is more frequent in CKD patients than in the general population (25). However, the causal relationship between CKD and PAD has not yet been fully elucidated. The prevailing opinion on this subject is that CKD is probably a marker of metabolic diseases leading to progressive dysfunction of vascular structure $(32,33)$. This suggestion is mostly based on studies that have investigated the association of atherosclerosis and albuminuria that is a marker of endothelial dysfunction (34). The association between albuminuria, medial arterial calcification and PAD is well established $(35,36)$. In addition to the direct impact of CKD on arteries, the risk factors for both CKD and PAD development are quite similar, though not identical $(37,38)$. In the Chronic Renal Insufficiency Cohort study, novel risk factors associated with an increased prevalence of PAD in patients with CKD were oxidative stress, inflammation, insulin resistance, and a prothrombotic state (39).

This study has some limitations. First, this was a retrospective analysis of a dataset created to explore the association of $\mathrm{ABI}$ and bone mineral density (19). Therefore, the study may be underpowered to study the relationship between severely reduced kidney functions and $A B I$ or peripheral arterial disease. Second, we calculated eGFR by using serum creatinine level at study entry using the MDRD study equation, which requires stable kidney function (39). Although the participants were community-dwelling outpatients, the current study did not specifically assess the course of kidney functions for at least three months. Third, it is well-known that the prevalence of PAD is significantly higher among dialysis patients (25) and end-stage CKD patients (36). However, we excluded stage 5 CKD patients and patients receiving renal replacement therapy, which might have caused a similar distribution of PAD between lower and higher eGFR groups after adjustment for confounders.

\section{Conclusion}

In conclusion, this study showed evidence of a higher prevalence of PAD in older people with CKD. However, the observed difference appeared to depend on existing confounders in multivariable regression analyses, suggesting that the risk of PAD in older adults with CKD is not modified independently from other risk factors for atherosclerotic diseases. Based on the findings in our study and current literature, future prospective studies with larger sample size must better identify the association of mild to moderate CKD with PAD among older adults.

\section{Ethics}

Ethics Committee Approval: The Kecioren Training and Research Hospital Institutional Review Board approved the study (approval number: 2012-KAEK-15/1256-2017, date: 11.01.2017). 
Informed Consent: This study is an analysis of a data set from a prospective study.

Peer-review: Externally and internally peer-reviewed.

\section{Authorship Contributions}

Concept: İ.T., Design: I.T., Data Collection or Processing: B.B.B., I.T., Analysis or Interpretation: B.B.B., I.T., Literature Search: B.B.B., I.T., Writing: B.B.B., I.T.

Conflict of Interest: No conflict of interest was declared by the authors.

Financial Disclosure: The authors declared that this study received no financial support.

\section{References}

1. Grundy SM, Stone NJ, Bailey AL, et al. $2018 \mathrm{AHA} / \mathrm{ACC} /$ AACVPR/AAPA/ABC/ACPM/ADA/AGS/APhA/ASPC/NLA/ PCNA Guideline on the Management of Blood Cholesterol: Executive Summary: A Report of the American College of Cardiology/American Heart Association Task Force on Clinical Practice Guidelines. Circulation. 2019;139:1046-1081.

2. Tasci I. Comment on: Hanssen et al. Associations between the ankle-brachial index and cardiovascular and all-cause mortality are similar in individuals without and with type 2 diabetes: nineteen-year follow-up of a population-based cohort study. Diabetes Care 2012;35:1731-1735. Diabetes Care. 2013;36:25-26.

3. Tasci I, Kabul HK. Ankle-brachial index as an indicator of arterial stiffness. Angiology. 2012;63:155.

4. Fowkes FG. Epidemiology of atherosclerotic arterial disease in the lower limbs. Eur J Vasc Surg. 1988;2:283-291.

5. Selvin E, Erlinger TP. Prevalence of and risk factors for peripheral arterial disease in the United States: results from the National Health and Nutrition Examination Survey, 19992000. Circulation. 2004;110:738-743.

6. Bozkurt AK, Tasci I, Tabak O, Gumus M, Kaplan Y. Peripheral artery disease assessed by ankle-brachial index in patients with established cardiovascular disease or at least one risk factor for atherothrombosis--CAREFUL study: a national, multicenter, cross-sectional observational study. BMC Cardiovasc Disord. 2011;11:4.

7. Tasci I, Safer U, Naharci MI, et al. Undetected Peripheral Arterial Disease Among Older Adults With Alzheimer's Disease and Other Dementias. Am J Alzheimers Dis Other Demen. 2018;33:5-11.

8. Hirsch AT, Haskal ZJ, Hertzer NR, et al. ACC/AHA 2005 Practice Guidelines for the management of patients with peripheral arterial disease (lower extremity, renal, mesenteric, and abdominal aortic): a collaborative report from the American Association for Vascular Surgery/Society for Vascular Surgery, Society for Cardiovascular Angiography and Interventions, Society for Vascular Medicine and Biology, Society of Interventional Radiology, and the ACC/AHA Task Force on Practice Guidelines (Writing Committee to Develop Guidelines for the Management of Patients With Peripheral Arterial Disease): endorsed by the American Association of Cardiovascular and Pulmonary Rehabilitation; National
Heart, Lung, and Blood Institute; Society for Vascular Nursing; TransAtlantic Inter-Society Consensus; and Vascular Disease Foundation. Circulation. 2006;113:463-654.

9. Norgren L, Hiatt WR, Dormandy JA, et al. Inter-Society Consensus for the Management of Peripheral Arterial Disease (TASC II). J Vasc Surg. 2007;45(Suppl S):5-67.

10. Verim S, Taşçı I. Doppler ultrasonography in lower extremity peripheral arterial disease. Turk Kardiyol Dern Ars. 2013;41:248-255.

11. Tekgöz E, Bozkurt E, Sağlam K. Assessment of association between ankle-brachial pressure index and pulse wave velocity in patients with isolated hypertension according to gender. Gulhane Med J. 2021;63:218-224.

12. Başgöz BB, Taşcı I, Yıldız B, Demirbaş Ş, Sağlam K, Açıkel C. Sensitivity, specificity and predictive value of the Edinburgh Claudication Questionnaire versus Ankle-Brachial Index for the diagnosis of lower extremity Arterial Disease in Turkish adults. Gulhane Med J. 2016;58:177-183.

13. Mallappallil M, Friedman EA, Delano BG, McFarlane SI, Salifu MO. Chronic kidney disease in the elderly: evaluation and management. Clin Pract (Lond). 2014;11:525-535.

14. Abdulkader RCRM, Burdmann EA, Lebrão ML, Duarte YAO, Zanetta DMT. Aging and decreased glomerular filtration rate: An elderly population-based study. PLoS One. 2017;12:e0189935.

15. Coresh J, Astor BC, Greene T, Eknoyan G, Levey AS. Prevalence of chronic kidney disease and decreased kidney function in the adult US population: Third National Health and Nutrition Examination Survey. Am J kidney Dis. 2003;41:1-12.

16. Garg AX, Clark WF, Haynes RB, House AA. Moderate renal insufficiency and the risk of cardiovascular mortality: results from the NHANES I. Kidney Int. 2002;61:1486-1494.

17. Shlipak MG, Fried LF, Crump C, et al. Cardiovascular disease risk status in elderly persons with renal insufficiency. Kidney Int. 2002;62:997-1004.

18. Leskinen $Y$, Salenius JP, Lehtimäki T, Huhtala $H$, Saha $H$. The prevalence of peripheral arterial disease and medial arterial calcification in patients with chronic renal failure: requirements for diagnostics. Am J kidney Dis. 2002;40:472-479.

19. Tasci I, Basgoz BB, Ince S, Safer U. Frequency of Peripheral Arterial Disease in Turkish Older Adults with and without Osteoporosis. JAGS. 2018;66(S2):S1-S369-(A221). Available from: https://meeting.americangeriatrics.org/sites/default/ files/inline-files/2018-Journal_of_the_American_Geriatrics_ Society.pdf

20. Levey AS, Bosch JP, Lewis JB, Greene T, Rogers N, Roth D. A more accurate method to estimate glomerular filtration rate from serum creatinine: a new prediction equation. Modification of Diet in Renal Disease Study Group. Ann Intern Med. 1999;130:461-470.

21. Basgoz BB, Tasci I, Yildiz B, Acikel C, Kabul HK, Saglam K. Evaluation of self-administered versus intervieweradministered completion of Edinburgh Claudication Questionnaire. Int Angiol. 2017;36:75-81.

22. Tasci I, Kabul HK. Diagnosing PAD by calculating the ankle brachial index: adherence to the guidelines. J Vasc Nurs. 2012;30:3-4. 
23. Kabul HK, Aydogdu A, Tasci I. Calculation methods of ankle brachial index and correct diagnosis of peripheral arterial disease. J Atheroscler Thromb. 2012;19:691-692.

24. Keith DS, Nichols GA, Gullion CM, Brown JB, Smith DH. Longitudinal follow-up and outcomes among a population with chronic kidney disease in a large managed care organization. Arch Intern Med. 2004;164:659-663.

25. O'Hare AM, Glidden DV, Fox CS, Hsu CY. High prevalence of peripheral arterial disease in persons with renal insufficiency: results from the National Health and Nutrition Examination Survey 1999-2000. Circulation. 2004;109:320-323.

26. Wattanakit K, Folsom AR, Selvin E, Coresh J, Hirsch AT, Weatherley BD. Kidney function and risk of peripheral arterial disease: results from the Atherosclerosis Risk in Communities (ARIC) Study. J Am Soc Nephrol. 2007;18:629-636.

27. Muntner P, He J, Hamm L, Loria C, Whelton PK. Renal insufficiency and subsequent death resulting from cardiovascular disease in the United States. J Am Soc Nephrol. 2002;13:745-753.

28. de Vinuesa SG, Ortega M, Martinez $P$, Goicoechea $M$, Campdera FG, Luño J. Subclinical peripheral arterial disease in patients with chronic kidney disease: prevalence and related risk factors. Kidney Int Suppl. 2005;93(Suppl):44-47.

29. Manjunath G, Tighiouart H, Coresh J, et al. Level of kidney function as a risk factor for cardiovascular outcomes in the elderly. Kidney Int. 2003;63:1121-1129.

30. Selvin E, Köttgen A, Coresh J. Kidney function estimated from serum creatinine and cystatin $C$ and peripheral arterial disease in NHANES 1999-2002. Eur Heart J. 2009;30:1918-1925.

31. Hirsch AT, Criqui $\mathrm{MH}$, Treat-Jacobson D, et al. Peripheral arterial disease detection, awareness, and treatment in primary care. JAMA. 2001;286:1317-1324.
32. Wu CK, Yang CY, Tsai CT, et al. Association of low glomerular filtration rate and albuminuria with peripheral arterial disease: the National Health and Nutrition Examination Survey, 19992004. Atherosclerosis. 2010;209:230-234.

33. Wattanakit K, Folsom AR, Criqui $\mathrm{MH}$, et al. Albuminuria and peripheral arterial disease: results from the multi-ethnic study of atherosclerosis (MESA). Atherosclerosis. 2008;201:212216.

34. Barzilay JI, Peterson D, Cushman M, et al. The relationship of cardiovascular risk factors to microalbuminuria in older adults with or without diabetes mellitus or hypertension: the cardiovascular health study. Am J Kidney Dis. 2004;44:25-34.

35. Aboyans V, Ho E, Denenberg JO, Ho LA, Natarajan L, Criqui $\mathrm{MH}$. The association between elevated ankle systolic pressures and peripheral occlusive arterial disease in diabetic and nondiabetic subjects. J Vasc Surg. 2008;48:1197-1203.

36. Jaar BG, Plantinga LC, Astor BC, et al. Novel and traditional cardiovascular risk factors for peripheral arterial disease in incident-dialysis patients. Adv Chronic Kidney Dis. 2007;14:304-313.

37. Rajagopalan S, Dellegrottaglie S, Furniss AL, et al. Peripheral arterial disease in patients with end-stage renal disease: observations from the Dialysis Outcomes and Practice Patterns Study (DOPPS). Circulation. 2006;114:1914-1922.

38. Chen J, Mohler ER, Xie D, et al. Risk factors for peripheral arterial disease among patients with chronic kidney disease. Am J Cardiol. 2012;110:136-141.

39. O'Hare A, Johansen K. Lower-extremity peripheral arterial disease among patients with end-stage renal disease. J Am Soc Nephrol. 2001;12:2838-2847. 